\title{
Clinical, Molecular and Histopathological Evaluation of the Effect Autologus PRP \& PRF on the Second Intension Wound Healing in Dogs
}

Olla Khalifa ( $\sim$ abdelhaleem_elkasapy@yahoo.com )

Benha University https://orcid.org/0000-0002-2783-6962

Abdelhaleem Elkasapy

Benha University

Eman Sallam

Benha University

Adel Alakraa

Benha University Faculty of Veterinary Medicine

Yasmin Marie

Benha University Faculty of Medicine

liza mohammed

Benha University Faculty of Veterinary Medicine

\section{Research Article}

Keywords: Wound, PRP, PRF, Gene expression

Posted Date: February 22nd, 2021

DOl: https://doi.org/10.21203/rs.3.rs-179329/v1

License: (9) (i) This work is licensed under a Creative Commons Attribution 4.0 International License. Read Full License 


\section{Abstract}

A new trend of regenerative medicine was introduced recently in the field of second intension wound healing. The PRP and PRF represented a corner stone in this clinical practice, were it admit many factors for acceleration of wound healing, enhancement of wound epithelization and neovascularization. PRP and PRF are considered as a great concentrated source of growth factors (PDGF, TGF- $\beta$, VEGF, bFGF) and cytokines which are fundamental fro wound healing.

Nine adult dogs were used in this study; we induced a $3 \mathrm{~cm}$ diameter full thickness cutaneous injury at the right chest region. The animals were divided into three groups equally and the wounds were treated twice weekly for three successive weeks. Group A received only normal saline (control group). The members of second group treated by PRP (group B) while, the group C treated by PRF. Clinical evaluation, Molecular studies of IL10 \& TGF- $\beta$ and histopathological examination were used to demonstrate the difference between the three treatment regimes. Results showed non-significant negative low correlation between loss of weight and WHR\%, and showed significant high positive correlation between treatment cost either by PRP or PRF with IL10 (0.79*) and WHR\%(0.996**).

We concluded that the PRP and PRF exhibited higher regeneration capacity and accelerate the quality of wound healing. The PRP was more superior to PRF, but not significantly different. The IL10 was significant increase in expression in PRP, while TGF-b was non-significant increased in PRF group

\section{Introduction}

The second intention wound healing represents a major problem in the veterinary practice, as it is more susceptible to infection with multidrug-resistant strains of pathogenic microorganism and dryness. The healing process begins directly from the boundaries of the wounds after the occurrence of the skin injury. The blood clot also acts as a bridge for cell migration. The blood plug replaced by granulation tissue three to five days later ushered in a start of re-epithelization [1].

Wound healing is process include four overlapping remodeling [2], involving numerous inflammatory mediators, immune cells, extracellular matrix (ECM), and parenchymal cells [3]. It begins with the homeostasis/inflammatory response, the platelet aggregation and production of numerous cytokines. In the proliferation process, occur migration and proliferation of keratinocytes, endothelial cells, and fibroblasts. And the recovery of the dermis occur after invading and proliferating by fibroblasts which considered as significantly important for the process of wound healing [2]. Tissue remodeling phase includes the newly formed capillaries and collagen reorganization. Collagen produced by fibroblasts and play an important role in each of these phases [4].

Platelets exhibited effective antimicrobial activity which improved the infected wound healing reepithelization and granulation tissue formation [5]. Platelets are a great source of wound healing agents as platelet-derived growth factor (PDGF), transforming growth factor- $\beta$ (TGF- $\beta$ ), vascular endothelial 
growth factor (VEGF) and platelet-derived angiogenesis factor (PDAF) [6], so increasing their concentration in the affected area enhance, reinforce and accelerate the healing process [7].

PRP (platelet-rich plasma) provide 2 of the 3 components (i.e., growth factors and scaffold) necessary to support true tissue regeneration and improvement significantly the healing of complex wounds[8].

PRF (platelet-rich fibrin) belongs to a new generation of platelet concentrates, with simplified processing, and without biochemical blood handling. PRF contains concentrated growth factors (PDGF,TGF- $\beta$,VEGF and basic fibroblast growth factor (bFGF)) $[9,10]$.

IL-10 (interleukin-10) is a homodimeric cytokine produced by a variety of cell types, including T cells, monocytes, and macrophages. In the skin, keratinocytes produce IL-10 after injury [11], activate macrophage/ monocyte functions, decrease pro-inflammatory cytokine production and regulate fibrogenic cytokines, such as (TGF-b) as a part of its role in the regulation of tissue remodeling $[12,13]$.

Wound infiltrated by M2 activated macrophages secreted TGF- $\beta[14,15]$, which stimulates migration and proliferation of fibroblasts at the wound side [16].

TGF- $\beta$ have both anti-inflammatory activity $[17,18]$, and pro-inflammatory effects [19], with regard that pro-inflammatory effects are predominant $[20,21]$.It have been reported that persistent inflammatory infiltrates in chronic ulcers contribute to TGF- $\beta$ levels $[19,22]$.

Our work aimed to: evaluate and comparison the effect of PRP and PRF on the second intention wound healing and the role of IL10 \& TGF- $\beta$ gene expression.

\section{Materials \& Methods}

\section{Experimental Animals}

The study was conducted on nine dogs aged 2-3 years and weighing $12-15 \mathrm{Kg}$. Under the influence of general anesthesia [23], acute full thickness skin wound of $3 \mathrm{~cm}$ diameter was made on the right side of the vertebral column in the chest area.

\section{Study Groups:}

The dogs were divided into three groups each one include three dogs, the wounds of all groups treated twice weekly for three successive weeks.

Group A (control group): the wounds of this group were subjected only to normal saline.

Group B (PRP treatment): the wounds were treated by autologous PRP where the edges of the wound were well covered, as was the wound itself. 
Group C (PRF treatment): the wounds were treated by autologous PRF, by the same technique adopted in the second group.

Then leave the wounds of all groups to heal with the second intention.

\section{Preparation of PRP by double centrifugation}

The blood obtained from the dogs was put into biochemistry tubes containing citrate-phosphatedextrose solution and centrifuged for $10 \mathrm{~min}$ at $1500 \mathrm{rpm}$. After centrifugation, 3 layers were obtained. The plasma layer at the top was collected in another centrifugation tube and subjected to a second centrifugation for $10 \mathrm{~min}$ at $3000 \mathrm{rpm}$, after which 2 layers were obtained; the upper layer was plateletpoor plasma (PPP) and the lower was PRP [24]. This final form of liquid PRP was applied to the wounds of the dogs.

\section{Preparation of PRF}

For each dog, the blood sample (1 tube of $15 \mathrm{ml}$ each) was obtained from a jugular vein. The blood samples were taken without anticoagulant in dry glass tubes and were immediately centrifuged at 3,000 rpm for 10 minutes with a specific table centrifuge at room temperature. After centrifugation, the PRF clot was removed from the tube using sterile tweezers, separated from the RBC base using sterile scissors, and placed in a sterile cup [25].

The evaluation and comparison between the three treatment regimens was carried out through clinical vision, real time quantitative PCR and histopathological examination.

\section{Evaluation of wound contraction rates}

Clinical evaluation achieved through digital photographs which taken from wounds in the presence of ruler for measuring of wound healing rate at days $0,7,14$ and 21 after injury. The wound healing rate in control, PRP and PRF-treated wounds was calculated according to the following equations:

WHR\% $=(\mathrm{iWA}-\mathrm{fWA}) / \mathrm{iWA} \times 100[26]$.

\section{Real time quantitative PCR}

Skin section will be taken immediately from anaesthetized animals and placed in Cryo tubes and stored in RNA later solution (by $10 \mu \mathrm{L}$ per $1 \mathrm{mg}$ of tissue) (Qiagen-GmbH, Germany) at- $80^{\circ} \mathrm{C}$.

Total RNA was isolated using Easy Red TM kit (Intron Biotechnology, Korea) according to the instructions of the manufacturer, it was eluted in nuclease-free water, purity quantified spectrophotometrically at 260 and $280 \mathrm{~nm}$, and kept at $-80^{\circ} \mathrm{C}$ until use. Ten $\mu \mathrm{L}$ RNA $(2 \mu \mathrm{g})$ from each sample were taken for Synthesis of cDNA using RT2First Strand Kit (Qiagen-GmbH, Germany) according to the manufacturer instruction. 
Quantitative Real Time PCR amplifications were performed with RT2 SYBR Green master mix (Qiagen$\mathrm{GmbH}$, Germany), in the thermocycler Real time PCR (Applied Biosystem 7500 Fast Real time PCR,USA), under the following cycle conditions: $95^{\circ} \mathrm{C}$ for 10 min followed by 40 cycles of $95^{\circ} \mathrm{C}$ for $15 \mathrm{sec}$ then $60^{\circ} \mathrm{C}$ for $1 \mathrm{~min}$.

The fold-change for each gene calculated using the formula: 2(- $\Delta \Delta C T)$ [27]. The $p$ values are calculated based on a Student's $t$ test of the replicate $2^{\wedge}$ (-Delta Ct) values for each gene in the control group and treatment groups and $p$ values less than 0.05 . Transcript quantities were normalized by RPS- 5 as a house keeping gene. The following primers (Invitrogen, Thermo Fisher Scientific, USA) were used for cDNA amplification (table 1).

Table 1 List of primer sequence.

\begin{tabular}{|ll|}
\hline Target gene & Sequence 5-3 \\
\hline RPS-5 & F: tcactggtgagaacccct R: cctgattcacacggcgtag [28]. \\
\hline IL-10 & F: cccgggctgagaaccacgac R: aaatgcgctcttcacctgctccac [29]. \\
TGF- $\beta$ & F: caaggatctgggctggaagtgga R: ccaggaccttgctgtactgcgtg [30]. \\
\hline
\end{tabular}

\section{Histopathological evaluation}

Specimens for histopathological evaluation were obtained on day 21 after surgery; the specimens were fixed in $10 \%$ neutral buffered formalin. After $24 \mathrm{~h}$ of fixation, the skin tissues were gradually dehydrated and embedded in paraffin. Then, a microtome was used to cut the tissues into $5-\mu m$-thick sections.

Paraffin sections were stained by Hematoxylin and Eosin (H\&E) for routine light microscopic examination and Masson's trichrome stain to detect connective tissue maturation. The depth and organization of the green color can differentiate the maturity of collagen fibers [31]. The degree of collagen production, cellular infiltration, neovascularization, and re-epithelialization process including thickness of epithelium over the wound are the parameters involved in wound healing. Histopathological evaluation of these parameters allowed the detection of differences between control and treated groups.

\section{Productive and economic measures}

Body weight was recorded individually for each dog in each group at beginning and at the end of the experiment

Treatment cost was defined as cost of inputs used to treat disease [32]: 
Control group receive only dressing (cost of dressing per day * number of days for each animal.

PRP receive dressing + PRP (PRP cost per day * number of days) for each animal.

PRF receive dressing + PRF (PRF cost per day * number of days) for each animal.

\section{Statistical analysis:}

The collected data in the present investigation are subjected to statistical analysis using one way analysis of variance (ANOVA) used to determine, wound dimensions, means of different variables as TGF, IL10, body weight changes and treatment cost. Correlation matrix was done to determine relationship between variables as body weight changes and treatment cost on TGF, IL10 and WHR \% .Linear regression: The regression test used to determine the best way of the correlations between WHR\%, TGF and IL 10 (dependent variable) with loss of weight and treatment cost (independent variable) the model of the functions was the logarithmic model. ( $t$ test) used to determine the significance of each relationship . Also, the adjusted coefficient of determination $\left(\mathrm{R}^{-2}\right)$ was calculated to determine the degree of each relationship between dependent and independent variables [33].

\section{Results}

\section{Clinical findings}

The wounds of the control group (group A) exhibited sepsis and elevated center in 2 of induced injury, while the last one showed dryness, thin fissuring of the blood blug during 2nd week. The PRP and PRF groups showed no significant difference in wound healing between them but the two groups showed a significant decrease between them an $d$ the animals of control group. The criteria of wound healing in group $B$ and $C$ run in speed more than control group (table, 2) 
Table 2

Clinical evaluation (wound dimension and wound healing rate percent of the three groups.

\begin{tabular}{|llll|}
\hline & 1st week & 2nd week & 3rd week \\
\hline Wound dimensions & & & \\
Control & $26.83 \pm 0.166$ & $21.33 \pm 0.33$ & $15.33 \pm 0.33$ \\
PRP & $22.5 \pm 0.289$ & $10.33 \pm 0.33$ & $0.7 \pm 0.166$ \\
PRF & $23.5 \pm 0.289$ & $11.67 \pm 0.33$ & $2.33 \pm 0.33$ \\
Wound healing rate \% & & & \\
Control & 10.7 & 29 & 49 \\
PRP & 25 & 65.7 & 97.7 \\
PRF & 21.7 & 61.3 & 92.3 \\
\hline
\end{tabular}

The PRP and PRF groups were significantly $(\mathrm{p} \otimes 0.05)$ lower than control group at 1 st to 3rd weeks

\section{Expression of IL10 \& TGF- $\beta$ in canine treated with PRP \& PRF}

Quantitative RT-PCR was performed to measure mRNA expression of IL10 \& TGF- $\beta$ in dogs with second intension wound followed by treatment with PRP \& PRF to asses healing.

There was significant up regulation in expression of IL 10 in experimental groups (PRP and PRF) with significant increase in expression of IL10 in PRP group than PRF group $(P<0.05)$ (Fig. 1a). Also there was up regulation in TGF-b expression in PRF group than PRP group but it was non-significant $(P>0.05)$ (Fig. 1b).

\section{Histopathological Findings}

Histopathological results of wounds on Day 21 after surgery are corresponding to the final stage of wound healing. The re-epithelialization process, in all groups, was occurring, notably extending to the wound surface, and covered by scab tissue or keratin. Occasionally, the proliferated epidermal epithelium in control group showed focal areas of incomplete re-epithelialization, composed of granulation tissue admixed with variable numbers of lymphocytes, few macrophages and surrounded by one or several layers of keratinocytes (Fig. 2A). The PRP-treated cases showed higher re-epithelialization level in the healed wounds with rete ridges formation in underlying granulation tissue (Fig. 2B). The PRF-treated group showed complete re-epithelialization; however, the thickness of epithelium over the wound was lower when compared with that of the wounds treated with PRP (Fig. 2C), which indicated that reepithelialization in this group was much slower than that in the PRP-treated group. 
The rate of collagen production was demonstrated in dermis. The PRP-treated cases had the highest level of collagen deposition (Fig. 3B). Meanwhile, the PRF-treated wounds had moderate collagen deposition (Fig. 3C) compared with control group which had granulation tissue formation with minimal collagen deposition (Fig. $3 \mathrm{~A}$ ) in dermis of wounds. These results were confirmed by the Masson's trichrome staining of collagen where the green color of the PRP-treated wounds was the most intense, indicating highly mature collagen fibers (Fig. 4). However, the control group presented the highest number of new blood vessels with formation of granulation tissue characterized by fibroblasts and collagen perpendicular to new blood vessels (Fig. 3A). The number of new vessels of the control cases reduced in PRP and PRF-treated wounds (Fig. 5). The granulation tissue of the dermis was infiltrated by small aggregates of inflammatory cells mainly lymphocytes and macrophages. The number of inflammatory cells in PRP and PRF treated groups was higher than that observed in control group (Fig. 5).

Table 3

Changes of body weight and treatment cost among different treated groups.

\begin{tabular}{|c|c|c|c|c|}
\hline & Initial weight & Final weight & Loss of weight & Treatment cost \\
\hline & Mean \pm SE & Mean \pm SE & Mean \pm SE & Mean \\
\hline Control & $13.67^{a} \pm 0.33$ & $12.67^{a_{ \pm} 0.33}$ & $1.00^{a_{ \pm} 0.00}$ & 21.00 \\
\hline PRP & $14.00^{a_{ \pm}} \pm .58$ & $13.28^{a} \pm 0.53$ & $0.72 a_{ \pm 0.17}$ & 51.00 \\
\hline PRF & $13.67^{a_{ \pm}}{ }_{0.88}$ & $12.85^{a} \pm 0.85$ & $0.82^{a_{ \pm}} \pm 0.09$ & 45.00 \\
\hline
\end{tabular}

Means within the same column carrying same letters showed non significant effect $(P>0.05)$.

Table 4

Correlation between body weight changes, treatment cost, TGF, IL10 and WHR\% of different treated groups.

\begin{tabular}{|llllllll|}
\hline & $\begin{array}{l}\text { Initial } \\
\text { weight }\end{array}$ & $\begin{array}{l}\text { Final } \\
\text { weight }\end{array}$ & $\begin{array}{l}\text { Loss of } \\
\text { weight }\end{array}$ & $\begin{array}{l}\text { Treatment } \\
\text { cost }\end{array}$ & TGF & IL10 & WHR\% \\
\hline TGF & -.542 & -.430 & -.480 & 0.614 & 1 & 0.323 & 0.654 \\
\hline IL10 & -.334 & -.377 & 0.337 & $.792^{\star}$ & 0.323 & 1 & $.74^{\star}$ \\
\hline $\begin{array}{l}\text { WHR } \\
\%\end{array}$ & -.101 & -.076 & -.113 & $.994^{\star *}$ & 0.654 & $.74^{\star}$ & 1 \\
\hline
\end{tabular}

**. Correlation is significant at the 0.01 level (2-tailed).

*. Correlation is significant at the 0.05 level (2-tailed). 
Table 5

Regression between WHR\% and treatment cost due to different treated methods and weight loss.

\begin{tabular}{|ll|}
\hline Function & Log WHR\% $=-0.64+0.79$ log treatment cost -0.05 Log loss of weight \\
T & $(14.66(* *(28.41(* *(-0.87)$ \\
Sig & 0.0001 \\
F & 420 \\
$\mathrm{R}^{-2}$ & .99 \\
\hline
\end{tabular}

Table 6

Regression between TGF, IL10 and treatment cost due to different treated methods.

\begin{tabular}{|c|c|}
\hline Function & Log TGF $=2.87+2.19$ log treatment cost \\
\hline $\mathbf{T}$ & $(-2.48)(2.97(*$ \\
\hline sig & 0.02 \\
\hline $\mathbf{F}$ & 8.80 \\
\hline$R^{-2}$ & .49 \\
\hline Function & $\log \operatorname{IL} 10=3.99+3.00$ log treatment cost \\
\hline $\mathrm{T}$ & $(-2).(85 *(3.37)(*$ \\
\hline Sig & 0.01 \\
\hline $\mathrm{F}$ & 11.32 \\
\hline$R^{-2}$ & .56 \\
\hline
\end{tabular}

Results in table (3) indicated that non-significant changes of method of wound treatment on body weight changes with less weight loss in treated groups in comparison to control group. Regarding treatment cost ranged from LE 21 in control group to LE 51 in PRP treated groups.

Regarding correlation between body weight changes\& treatment cost and immunity represent in TGF, IL10 and WHR \% results in table (4) showed non-significant negative low correlation between loss of weight and WHR\%, and showed significant high positive correlation between treatment cost with IL10 (0.79*) and WHR\%(0.994**). There was significant high positive correlation between IL10 and WHR \% and nonsignificant high correlation between TGF and WHR \%.

The results in Table 5 revealed that the logarithmic function was significant $(P<0.05)$, adjusted $R^{-2}$ was 0.99 . The average elasticity of treatment cost was about $(+0.79)$ and average elasticity of loss of weight was about (-0.05). 
The results in Table 6 revealed that the logarithmic function was significant $(P<0.05)$ adjusted $R^{-2}$ was 0.49 in TGF and 0.56 for IL10. The average elasticity of treatment cost was about $(+2.19)$ for TGF, and average elasticity of treatment cost was about (+3) for IL10.

\section{Discussion}

The wound treatment with PRP had healed rapidly and completely within 3 weeks, without formation of exuberant granulation tissue and with minimal scarring. This is attributed to PRP used in the wound margins stimulate the production of excessive inflammatory wound mediators that provide a moist wound environment, thus increase the percentage of wound healing rate. These results coincide with previous studies [34].

In our study, the prepared PRP from autologous blood gave a good healing rate percent without sepsis. These results agree with that previously discussed [35]. Who endorsed that the autologous PRP has many advantage of eliminating immune reaction, antimicrobial effect.

Wound healing of the member of PRF group showed no significant difference from PRP treated animals but still lower in the healing percentage rate. Our results come in agreement with previous studies [36]. These reported that the liquid nature of the PRP increases the power of diffusion and invasion to the tissue, so the effect is faster than PRF which has a gel in nature.

Healing sites of the wounds take the same line with that previously mentioned [37]. Who discussed that the healing wounds were almost level and there were relatively advanced epithelium at the wound junction of the wounds in both PRP and PRF groups as it was thicker enough in PRP-treated group. The wounds junction was filled with dense collagen bundles as the inner walls of the wound edges were united at all. Rich neovascularisation was found on both groups but more abundant in PRP treated animals.

Regarding our results we found up regulation in expression of both IL 10 and TGF-b in experimental groups (PRP and PRF) this is in agreement with study reported that in the first 6 hours post wounding, there were an increase

in the gene expression of mediators of inflammation such as IL-10 and growth factors including TGF-a and beta [38].

During the early phase of wound healing TGF levels typically increased and is present at high levels at sites of skin injury [39], as this factor contributes to the recruitment of inflammatory cells, angiogenesis, collagen production, and wound remodeling [40].

From our results we noticed that there was significant increase in expression of IL10 in PRP group than PRF group $P$ value less than 0.05 , also there was up regulation in TGF-b expression in PRF group than PRP group but it was non-significant $P$ value more than 0.05 . Which indicate better healing in PRP group 
than PRF group with less scar formation. Also a significant high positive correlation between IL10 and WHR \% and non-significant high correlation between TGF and WHR \% as fetal skin wounding is characterized with blunted inflammatory immune response relative to that initiated from adult wounding [41]. The reduced presence of immune cells results in reduced levels of inflammatory cytokines and growth factors such as Tgf- $\beta 1$ [42]. Moreover increased expression of IL-10 have a role to heal wound regeneratively with scar less healing, as it attenuate the inflammatory response [43], acts as a regulator of the extracellular matrix [44], fibroblast cellular function [45], and endothelial progenitor cells (EPC) [46].

Regarding method of wound treatment had non-significant effect on body weight changes. Additionally correlation matrix, showed non-significant negative low correlation between loss of weight and WHR\%, and showed significant high positive correlation between treatment cost (Cost of different methods used in wound treatment) with IL10 $(0.79 *)$ and WHR \%( 0.996**). These results in agreement with [47] who found that non-significant changes on body weight changes and other clinical variables between control and PRP-treated groups and concerning, treatment cost as PRP in this experiment was the highest cost and resulted in better healing rate in agreement with [48] who indicated that PRP was suitable method used for wound healing.

Regarding, the logarithmic function between WHR, loss of weight and treatment cost (cost of different methods used in wound treatment) was significant $(P<0.001)$, and about $99 \%$ from the changes in WHR were attributed to loss in body weight and cost of different methods used in wound treatment. The average elasticity of treatment cost was about (+0.79), meaning that increasing treatment cost by about $1 \%$ resulted in increased WHR by $(0.79 \%)$ and average elasticity of loss of weight was about $(-0.05 \%)$, meaning that increasing loss of weight by $1 \%$ resulted in decrease WHR by $(0.05 \%)$. Concerning, the logarithmic function between cost of different methods used in wound treatment and TGF and between treatment cost \& IL10 was significant $(P<0.05)$, and about $49 \%$ from the changes in TGF and $56 \%$ from changes in IL 10 were attributed to changes in treatment cost due to different methods used in wound treatment. The average elasticity of treatment cost was about (+2.19), meaning that increasing treatment cost by about $1 \%$ resulted in increased TGF by $(2.19 \%)$ and average elasticity of treatment cost was about $(+3 \%)$, meaning that increasing treatment cost by about $1 \%$ resulted in increased IL10 by (3\%) this results indicated that treatment with either PRP and PRF resulted in wound healed more than normal dressing procedure as cost increased in PRP than PRF and the lowest in normal dressing this results in agreement with [49] who reported that PRP could be used for the treatment of all types of wounds and was in accordance of $[50,51]$.

\section{Conclusion}

From our clinical evaluation, molecular studies and histopathological examination we could conclude that the application of autologous PRP has better healing percentage rate than PRF. PRP accelerate wound healing through enhancement of wound re-epithelization, neovascularization and reduce scar formation. 
Research on the extended implications of TGF- $\beta$ signaling \& IL10 in wounds studied revealed significant increase in expression of IL10 in PRP group than PRF group. Also there was up regulation in TGF-b expression in PRF group than PRP group but it was non-significant.

\section{Declarations}

\section{Acknowledgment}

The authors extended their appreciation to the scientific research fund at Benha University this work

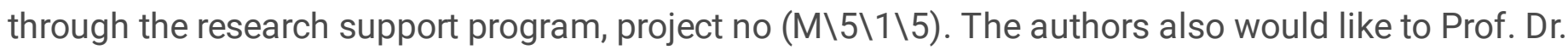
Shawky Moustafa for the histopathological examinations.

\section{Authors contributions:}

Conceptualization, design, supervision and corresponding author: Olla A. Khalifa.

Methodology, investigation, resources, data curation, writing original draft preparation, review and editing : Olla A. Khalifa, Abdelhaleem H. Elkasapy, Eman A. Sallam, Adel M .Alakraa, Yasmin M. Marei and Liza S. Mohammed. All authors have read and agreed to the published version of the manuscript.

Data Availability: the data used to support the findings of this study are included within the article.

\section{Compliance with Ethical Standards:}

All animal experiment processes were approved by Institutional Animal Care and Use Committee (IACUC), faculty of Veterinary Medicine Benha University, and it was strictly designed under the consideration of animal welfare.

Conflict of interest: The authors declare that they have no conflict of interest

Code availability: Not applicable

\section{References}

1. Farghali HA et al., Evaluation of subcutaneous infiltration of autologous platelet-rich plasma on skinwound healing in dogs. Bioscience reports, 2017. 37(2)

2. Singer AJ, Clark RA (1999) Cutaneous wound healing. New England journal of medicine 341(10):738-746

3. Bielefeld KA, Amini-Nik S, Alman BA (2013) Cutaneous wound healing: recruiting developmental pathways for regeneration. Cell Mol Life Sci 70(12):2059-2081

4. Kallis PJ, Friedman AJ (2018) Collagen Powder in Wound Healing. Journal of drugs in dermatology: JDD 17(4):403-408 
5. Bielecki T et al (2007) Antibacterial effect of autologous platelet gel enriched with growth factors and other active substances: an in vitro study. J Bone Joint Surg Br Vol 89(3):417-420

6. Nikolidakis D, Jansen JA (2008) The biology of platelet-rich plasma and its application in oral surgery: literature review. Tissue Engineering Part B: Reviews 14(3):249-258

7. Mishra A, Woodall J Jr, Vieira A (2009) Treatment of tendon and muscle using platelet-rich plasma. Clinics in sports medicine 28(1):113-125

8. Villela DL, Santos VLC (2010) Evidence on the use of platelet-rich plasma for diabetic ulcer: a systematic review. Growth factors 28(2):111-116

9. Kimura A et al (2005) The effects of platelet-rich plasma on cutaneous incisional wound healing in rats. J Dermatol Sci 40(3):205-208

10. Dohan DM et al., Platelet-rich fibrin (PRF): a second-generation platelet concentrate. Part I: technological concepts and evolution. Oral Surgery, Oral Medicine, Oral Pathology, Oral Radiology, and Endodontology, 2006. 101(3): p. e37-e44

11. An L et al., Effects of UVA on TNF-a, IL-1 $\beta$, and IL-10 expression levels in human keratinocytes and intervention studies with an antioxidant and a JNK inhibitor. Photodermatology, photoimmunology \& photomedicine, 2010. 26(1): pp 28-35

12. Moore KW et al (2001) Interleukin-10 and the interleukin-10 receptor. Annu Rev Immunol 19(1):683765

13. Fortunato SJ, Menon R, Lombardi SJ (1998) The effect of transforming growth factor and interleukin-10 on interleukin-8 release by human amniochorion may regulate histologic chorioamnionitis. American journal of obstetrics gynecology 179(3):794-799

14. Sato T et al (2010) Accelerated wound healing mediated by activation of Toll-like receptor 9 . Wound Repair Regeneration 18(6):586-593

15. Brancato SK, Albina JE (2011) Wound macrophages as key regulators of repair: origin, phenotype, and function. Am J Pathol 178(1):19-25

16. Werner S, Grose R, Regulation of wound healing by growth factors and cytokines. Physiological reviews, 2003

17. Shull MM et al (1992) Targeted disruption of the mouse transforming growth factor- $\beta 1$ gene results in multifocal inflammatory disease. Nature 359(6397):693-699

18. Kulkarni $\mathrm{AB}$ et al., Transforming growth factor beta 1 null mutation in mice causes excessive inflammatory response and early death. Proceedings of the National Academy of Sciences, 1993. 90(2): p. $770-774$

19. Gilbert RW, Vickaryous MK, Viloria-Petit AM (2016) Signalling by transforming growth factor beta isoforms in wound healing and tissue regeneration. Journal of developmental biology 4(2):21

20. Wang X-J et al (2006) Role of TGF $\beta$-mediated inflammation in cutaneous wound healing. in Journal of Investigative Dermatology Symposium Proceedings. Elsevier

21. Han G et al (2012) The pro-inflammatory role of TGFß1: a paradox? Int J Biol Sci 8(2):228 
22. Finnson KW et al (2013) Dynamics of transforming growth factor beta signaling in wound healing and scarring. Advances in wound care 2(5):195-214

23. Waelbers T, Vermoere P, Polis I (2009) Total intravenous anesthesia in dogs. Vlaams Diergeneeskundig Tijdschrift 78(3):160-169

24. Esat DM et al (2016) Comparison of the Effects of Plateletrich Plasma Prepared in Various Forms on the Healing of Dermal Wounds in Rats. Wounds: a compendium of clinical research practice 28(3):99

25. Dohan Ehrenfest DM et al (2010) Three-dimensional architecture and cell composition of a Choukroun's platelet-rich fibrin clot and membrane. Journal of periodontology 81(4):546-555

26. Eldebany $\mathrm{N}$ et al., Gelatin Loaded Titanium Dioxide and Silver Oxide Nanoparticles: Implication for Skin Tissue Regeneration. Biological Trace Element Research, 2020: p. 1-12

27. Livak KJ, Schmittgen TD (2001) Analysis of relative gene expression data using real-time quantitative PCR and the $2-\Delta \Delta C T$ method. methods 25(4):402-408

28. Brinkhof $B$ et al (2006) Development and evaluation of canine reference genes for accurate quantification of gene expression. Anal Biochem 356(1):36-43

29. Veenhof EZ et al (2010) Evaluation of T-cell activation in the duodenum of dogs with cutaneous food hypersensitivity. American journal of veterinary research 71(4):441-446

30. Spee B et al (2005) Regenerative and fibrotic pathways in canine hepatic portosystemic shunt and portal vein hypoplasia, new models for clinical hepatocyte growth factor treatment. Comp Hepatol 4(1):7

31. Bancroft JD, Gamble M, Theory and practice of histological techniques (2008) Elsevier health sciences

32. Bennett R, IJpelaar J (2005) Updated Estimates of the Costs Associated with Thirty Four Endemic Livestock Diseases in Great Britain: A Note. J Agric Econ 56(1):135-144

33. Spss I (2007) SPSS version 16.0. SPSS Incorporated, Chicago

34. Kazemi Mehrjerdi $\mathrm{H}$ et al (2008) Efficacy of autologous platelet-rich plasma (prp) activated by thromboplastin-D on the repair and regeneration of wounds in dogs. Iranian Journal of Veterinary Surgery 3(4):19-30

35. Farghali HA et al (2019) Antimicrobial action of autologous platelet-rich plasma on MRSA-infected skin wounds in dogs. Scientific reports 9(1):1-15

36. Tsai H-C et al (2019) A mini-pig model for evaluating the efficacy of autologous platelet patches on induced acute full thickness wound healing. BMC Vet Res 15(1):191

37. Khanzadeh Alishahi M et al (2014) Histopathological evaluation of the effect of platelet-rich fibrin on canine cutaneous incisional wound healing. Iranian Journal of Veterinary Science Technology $5(2): 19-32$

38. Chamorro Cl, Reinfeldt Engberg G, Fossum M (2020) Molecular and histological studies of bladder wound healing in a rodent model. Wound Repair Regeneration 28(3):293-306 
39. James LC et al (1991) Transforming growth factor a: In vivo release by normal human skin following UV irradiation and abrasion. Skin Pharmacology Physiology 4(2):61-64

40. Riedel $\mathrm{K}$ et al (2007) TGF- $\beta$ antisense therapy increases angiogenic potential in human keratinocytes in vitro*. Arch Med Res 38(1):45-51

41. Satish L, Kathju S, Cellular and molecular characteristics of scarless versus fibrotic wound healing. Dermatology research and practice (2010) 2010

42. Sullivan KM et al (1995) A model of scarless human fetal wound repair is deficient in transforming growth factor beta. Journal of pediatric surgery 30(2):198-203

43. Gordon A et al (2008) Permissive environment in postnatal wounds induced by adenoviral-mediated overexpression of the anti-inflammatory cytokine interleukin-10 prevents scar formation. Wound Repair Regeneration 16(1):70-79

44. King A et al (2013) Interleukin-10 regulates the fetal hyaluronan-rich extracellular matrix via a STAT3dependent mechanism. J Surg Res 184(1):671-677

45. Leung A et al (2013) Interleukin-10 and hyaluronan are essential to the fetal fibroblast functional phenotype. J Surg Res 179(2):257-258

46. Krishnamurthy $P$ et al (2011) Interleukin-10 deficiency impairs bone marrow-derived endothelial progenitor cell survival and function in ischemic myocardium. Circulation research 109(11):12801289

47. Abegão KGB et al (2015) Effects of heterologous platelet-rich plasma gel on standardized dermal wound healing in rabbits. Acta cirúrgica brasileira 30(3):209-215

48. Akhundov K et al (2012) Development of a cost-effective method for platelet-rich plasma (PRP) preparation for topical wound healing. Annals of burns fire disasters 25(4):207

49. Akhundov K et al (2012) Development of a cost-effective method for platelet-rich plasma (PRP) preparation for topical wound healing. Ann Burns Fire Disasters 25(4):207-213

50. Gaßling VL et al., Platelet-rich plasma and platelet-rich fibrin in human cell culture. Oral Surgery, Oral Medicine, Oral Pathology, Oral Radiology, and Endodontology, 2009. 108(1): p. 48-55

51. Bajaj P et al (2013) Comparative evaluation of autologous platelet-rich fibrin and platelet-rich plasma in the treatment of mandibular degree II furcation defects: a randomized controlled clinical trial. J Periodontal Res 48(5):573-581

\section{Figures}


(a)

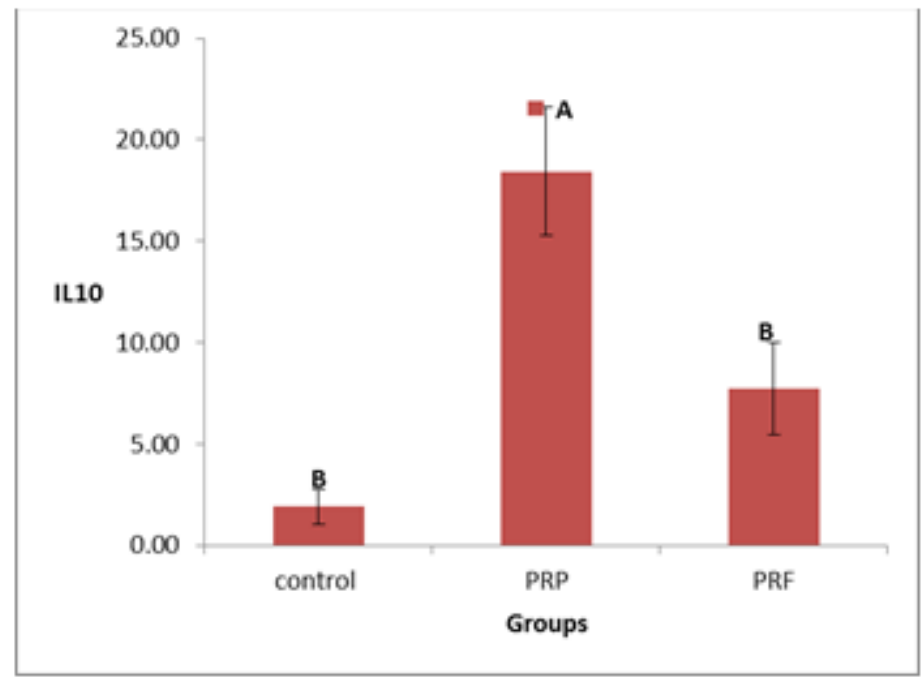

(b)

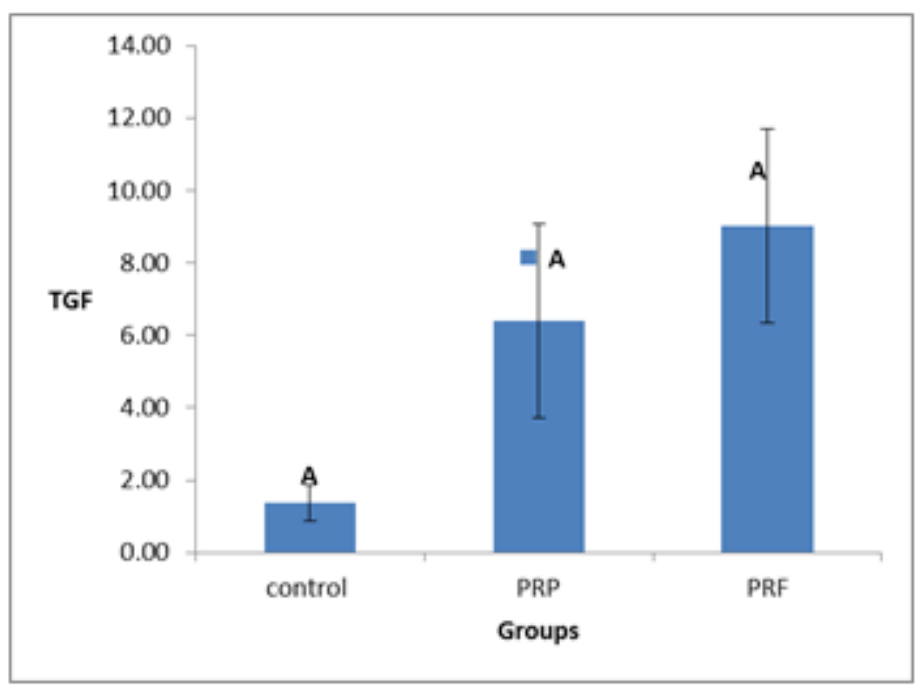

\section{Figure 1}

Relative m.RNA expression for (a) IL 10 and (b) TGF- $\beta$, it were determined by comparison with the value of control group and treated groups for each dog normalized against RPS- 5 as a house keeping gene, each bar represents the group mean +S.E.M.
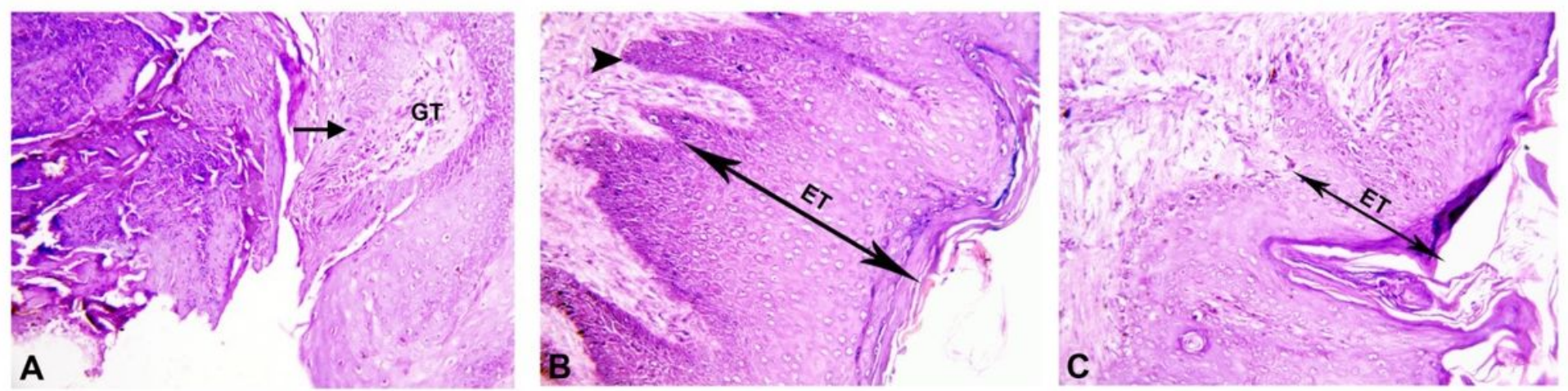

Figure 2 
Re-epithelialization of wound healing in dog on day 21 after-surgery (X20; H\&E stain): (A) Control group showing focal area of incomplete re-epithelialization, composed of granulation tissue (GT) admixed with inflammatory cells and surrounded by several layers of keratinocytes (arrow). (B) PRP-treated group showing higher re-epithelialization level (ET) with rete ridges formation (arrowhead). (C) PRF-treated group showing complete re-epithelialization with lower epithelium thickness (ET).
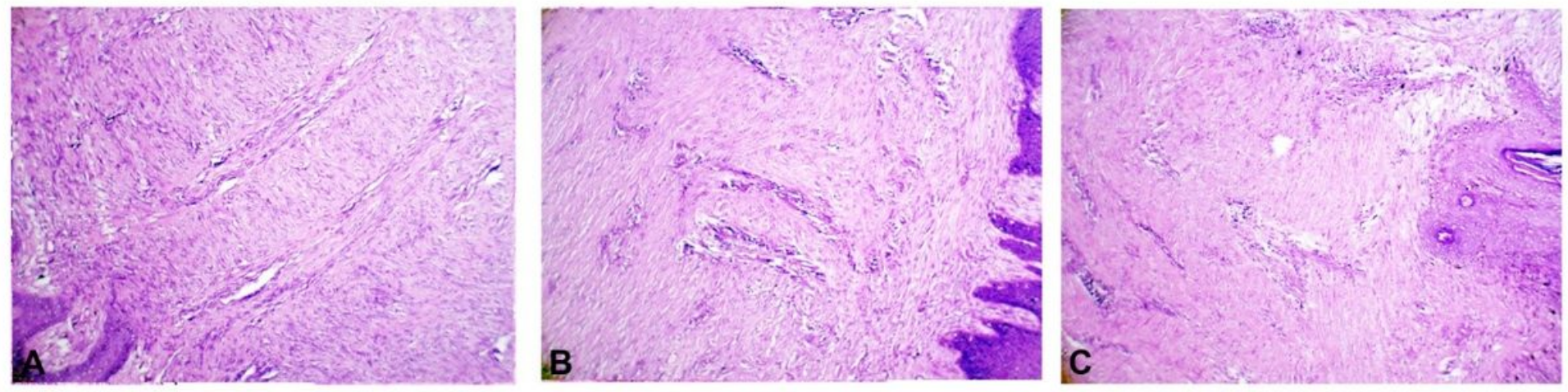

Figure 3

Collagen production in dermis of wound healing in dog on day 21 after -surgery (X10, H\&E stain): (A) Control group showing minimal collagen deposition. Note fibroblasts perpendicular to new blood vessels (B) PRP-treated group showing high level of collagen deposition. (C) PRF-treated group showing moderate collagen deposition.
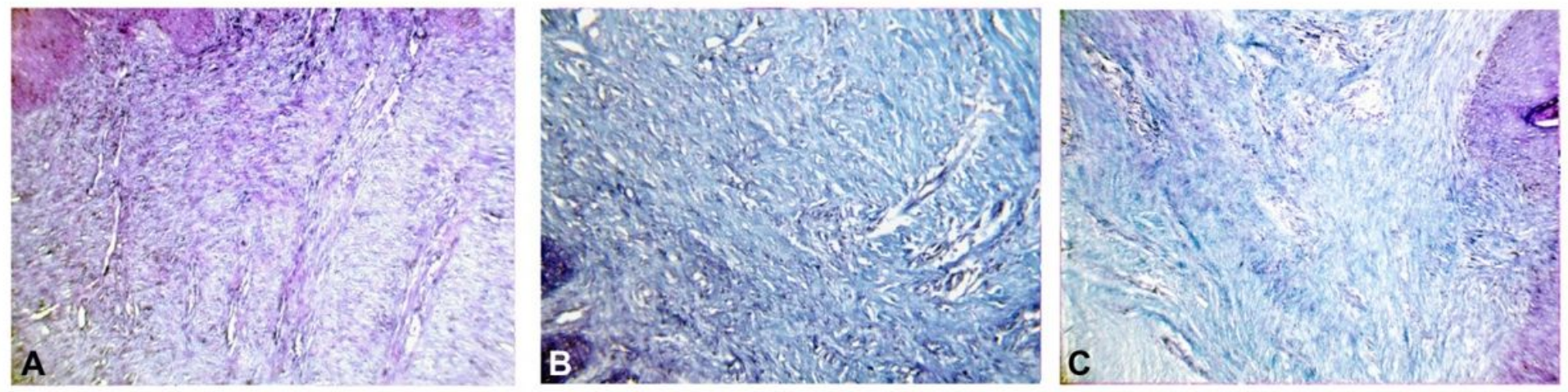

\section{Figure 4}

Masson's Trichrome staining of collagen production in wound healing in dog (X20). (A) Control group showing light green color of the immature collagen. B) PRP-treated group showing intense green color indicating highly mature collagen fibers. (C) PRF-treated group showing moderate green color of collagen. 

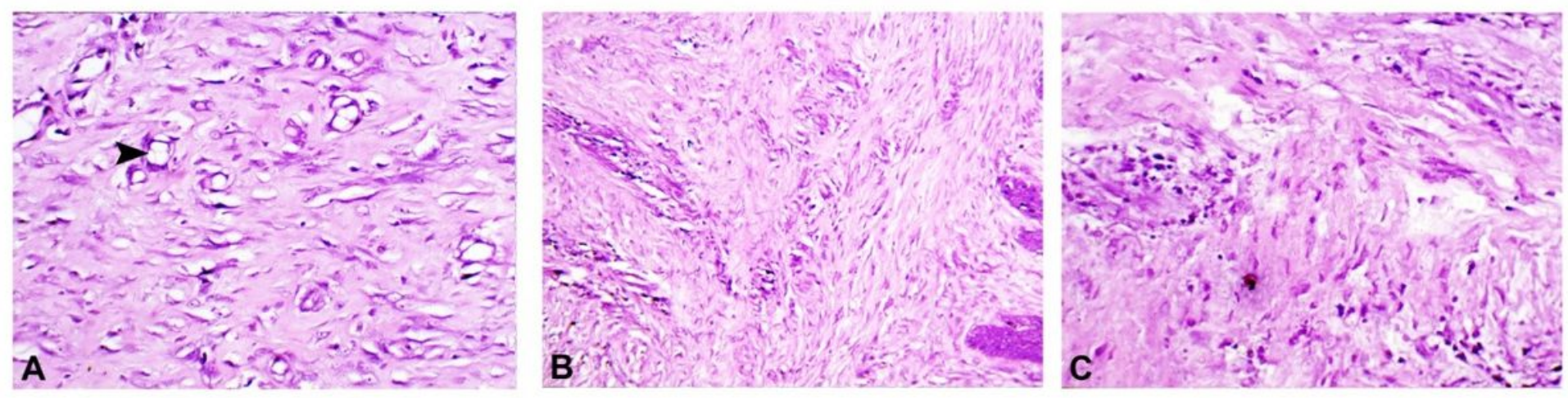

\section{Figure 5}

Neovascularization and inflammatory cellular infiltration in dermis of skin wound healing in dog on day 21 after-surgery (H\&E stain). (A) Control group showing high number of new vessels (arrowhead) and small number of inflammatory cells (X40). (B) PRP-treated group showing small number of new vessels and moderate number of inflammatory cells (X20). (C) PRF-treated group showing small number of new vessels and moderate number of inflammatory cells $(\mathrm{X} 40)$. 